\title{
Research on the Boundary Identification Method of Concealed Geological Bodies Based on Non-seismic Geophysical Data- Taking Benxi-Xiuyan Area as an Example
}

\author{
Mingli Yang ${ }^{1,2}$, Linfu Xue ${ }^{1 *}$, and Xiangjin $\operatorname{Ran}^{1}$ \\ ${ }^{1}$ College of Earth Science, Jilin University, 2199 Jianshe Street, Changchun, P.R. China \\ ${ }^{2}$ Engineering College, Jilin Business and Technology College, 1666 Kalun Lake Street, Changchun, P.R. China
}

\begin{abstract}
In the process of deep geological structure research, gravity and aeromagnetic anomaly data are often used to invert the distribution law of concealed geological bodies. In order to reveal the boundaries of concealed geological bodies and divide structural divisions, this paper adopts fuzzy control simulated annealing genetic algorithm () to superimpose the gravity and aeromagnetic anomaly data in Benxi-Xiuyan area and classify the patterns, and identify the main geological body boundaries and fractures. Comparing the relationship with known geological maps, the two have a higher degree of matching. Experiments show that the application of SAGAFcm algorithm can quickly identify the boundaries of concealed geological bodies and provide a new means for field geological mapping.
\end{abstract}

\section{Introduction}

With the development of regional geophysical work, research areas and deep geological structures, and the search and development of deep mineral resources have been paid more and more attention. Regional geological research and deep geological research need to make full use of regional gravity and aeromagnetic data to obtain deep geological information. The traditional analysis method is to process and interpret gravity and aeromagnetic data separately, and cannot intuitively and comprehensively use gravity and aeromagnetic data, and it is difficult to integrate regional geological data to perform comprehensive geological and geophysical analysis. Due to the different depths and physical properties of gravity and aeromagnetic methods, this paper proposes a method of superimposing gravity anomaly maps and aeromagnetic anomaly maps to comprehensively study the relationship between gravity and aeromagnetic anomalies and geological features. The SAGAFcm algorithm [1] is used to classify the superimposed information of gravity and aeromagnetic anomalies, divide the study area into areas with different combinations of gravity and aeromagnetic characteristics, and comprehensively analyse the relationship with regional geological data and regional mineral data to discover new important geological laws. Comprehensive analysis of gravity and aeromagnetic field characteristics can better reflect actual geological conditions, and research on the relationship with regional geological data and regional mineral geological data can reveal important geological laws.

\section{Regional geological background and petrophysical parameters}

\subsection{Regional geological background}

The Benxi-Xiuyan area is located at $123^{\circ}-124^{\circ} 30^{\prime}$ east longitude and $40^{\circ} 12^{\prime}-41^{\circ} 30^{\prime}$ north latitude. It is located in the north eastern part of the North China Craton, straddling the Longgang block and the Liaoji Paleoproterozoic rift. As shown in Fig. 1(a) [12].

The northern part is the Longgang block, which is the Archean ancient continental core [3], and mainly developed the Archean Anshan Group and plutonic intrusive rocks. A set of greenschist-amphibolite facies metamorphic rocks developed, which are the occurrence horizons of the famous Anshan-type BIF iron deposit [4]. The main body of Longgang block has Archean metamorphic basement and two-layer structure of Neoproterozoic Paleozoic sedimentary rock series. The southern margin of Longgang block also has three-layer structure of Paleoproterozoic Langzishan formation and Lieryu formation with smaller thickness

At present, the research area has completed 1:200,000, 1:250,000 regional geological surveys and 1:200,000 gravity and magnetic surveys. A large amount of regional geology, geophysics and other data have been obtained for the research area, laying a solid foundation for related research in the research area [5]. We obtained about 20,000 latitude and longitude points of gravity and aeromagnetic information as the test data for the experiment. 
Fig. 1(a) is a approximate location of the study area's study area. geotectonics, (b) is a simplified geological map of the
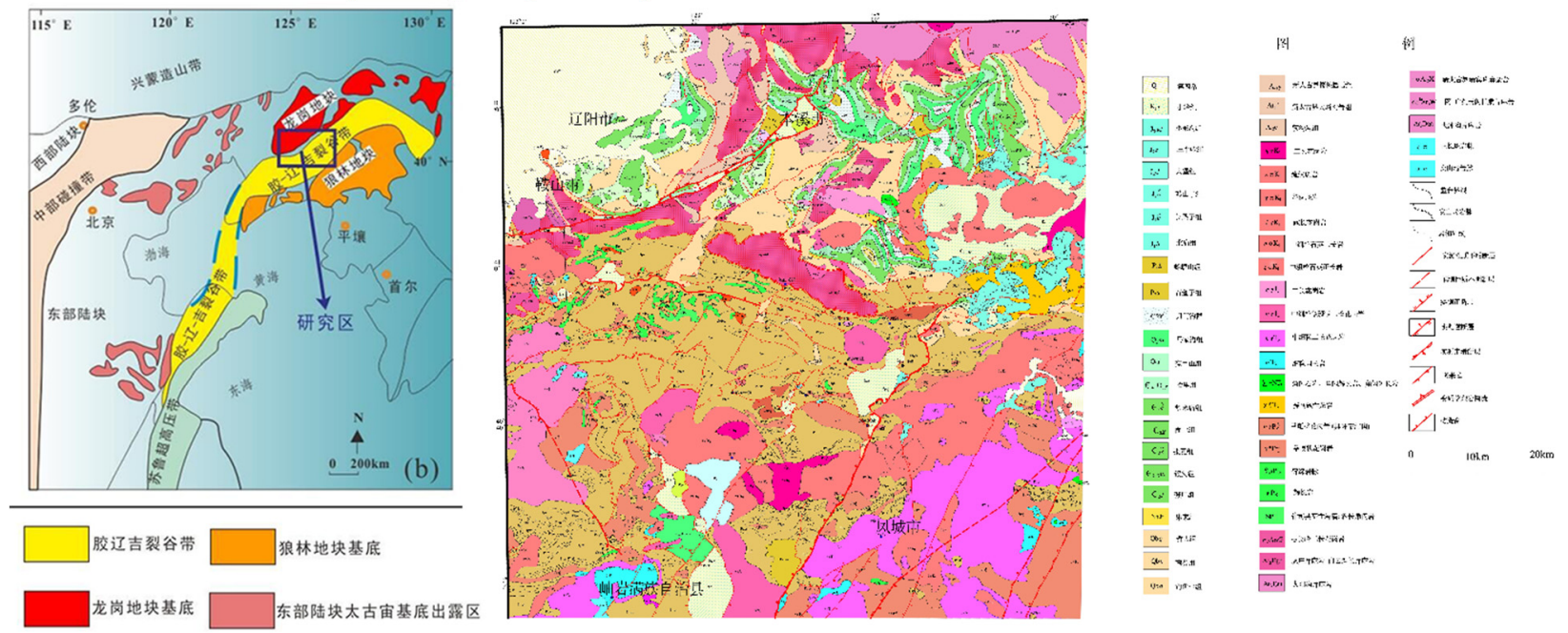

(a) Approximate location of the study area's geotectonics

(b) Geological sketch map of the study area

Figure 1. Tectonic location and geological sketch map of the study area

\subsection{Petrophysical parameters}

The stratum rock density of the strata in each age in the study area has a trend of gradually decreasing from old to new. The magnetic characteristics of the stratigraphic rocks are mainly that the Anshan Group, the Liaohe Group, and Mesozoic-Cenozoic volcanic rocks-volcanic sedimentary rocks have certain magnetic properties. The specific parameter characteristics are shown in Table 1. [6, 7]

Table 1. The petrophysical parameters of main rock masses in the study area

\begin{tabular}{|c|c|c|c|c|c|c|}
\hline \multirow{2}{*}{ Era } & \multirow{2}{*}{ Rock Mass } & \multirow{2}{*}{ Lithology } & \multicolumn{2}{|c|}{$\begin{array}{c}\text { Magnetic Susceptibility } \\
\left(10^{-5} \mathrm{SI}\right)\end{array}$} & \multicolumn{2}{|c|}{ Density $\left(\mathrm{g} / \mathrm{cm}^{3}\right)$} \\
\hline & & & $\begin{array}{l}\text { Change } \\
\text { Interval } \\
\end{array}$ & $\begin{array}{c}\text { Common } \\
\text { Value }\end{array}$ & $\begin{array}{l}\text { Change } \\
\text { Interval }\end{array}$ & $\begin{array}{c}\text { Common } \\
\text { Value }\end{array}$ \\
\hline \begin{tabular}{|c|} 
Early \\
Cretaceou \\
s \\
\end{tabular} & $\begin{array}{c}\text { Balidianzi Rock } \\
\text { Mass }\end{array}$ & $\begin{array}{l}\text { Medium-coarse } \\
\text { alkaline granite }\end{array}$ & 1004-2099 & 1689 & $2.53-2.64$ & 2.54 \\
\hline $\begin{array}{c}\text { Early } \\
\text { Jurassic }\end{array}$ & $\begin{array}{c}\text { anjialing Super } \\
\text { Unit British Room } \\
\text { Unit }\end{array}$ & $\begin{array}{l}\text { Medium-fine- } \\
\text { grained } \\
\text { monzonitic } \\
\text { granite }\end{array}$ & $99-215$ & 155 & $2.53-2.56$ & 2.55 \\
\hline $\begin{array}{c}\text { Paleoprot } \\
\text { erozoic }\end{array}$ & $\begin{array}{l}\text { Tongjiangyu } \\
\text { Complex }\end{array}$ & $\begin{array}{l}\text { Monzonitic } \\
\text { granite }\end{array}$ & $1-9$ & 1 & $2.50-2.66$ & 2.60 \\
\hline & & $\begin{array}{l}\text { Monzonitic } \\
\text { granite }\end{array}$ & $1-6$ & 1 & $2.55-2.58$ & 2.57 \\
\hline & & $\begin{array}{l}\text { Amphibolite } \\
\text { gneiss }\end{array}$ & $2-7$ & 1 & $2.50-2.59$ & 2.58 \\
\hline
\end{tabular}

\section{Pattern classification}

\subsection{Gravity and magnetic field pattern classification process}

- Preprocessing of gravity and aeromagnetic field data. The gravity and magnetic field can be preprocessed according to different geological interpretation targets. Generally, filtering or continuation methods can be used to process the gravity and aeromagnetic field. For eliminating the noise of the gravity Aeromagnetic field, we perform high-frequency filtering on the gravity aeromagnetic field. Use upward continuation to explore deep geological structures and conditions. The Aeromagnetic anomaly data should be processed to make the gravity anomaly have a good correspondence with the aeromagnetic anomaly.

- Gridding and normalization of gravity and aeromagnetic field data. The same grid parameters are used to grid the gravity and aeromagnetic field. At the same time, since the gravity value and the aeromagnetic value have different values and varying ranges, it is necessary to normalize the gravity data and the aeromagnetic data.

- Select the signs and group them. The original gravity and aeromagnetic data were selected by mark selection and grouping test, and the verification effect was observed and better grouping was selected for data cutting. 
- Aeromagnetic data cutting. Because when inputting as raw data, only gravity anomaly data and aeromagnetic anomaly data cannot be classified correctly, so this article intends to use linear interpolation cutting method to cut the aeromagnetic data as input data.

- Pattern classification. The pattern classification method is used to comprehensively classify the gravity field. Different weighting factors can be set in the classification process to determine the weight of the gravity field and magnetic field in the pattern classification process. Determine the number of categories.

- Summarize the characteristics of various types of gravity magnetic field, and renumber the gravity magnetic field types according to certain rules.

- Draw the model classification results map, and perform overlay analysis with the regional geological map to find out the relationship between the type of gravity and magnetic field and geological features and geological elements.

The specific process of pattern classification is shown in Figure 2.

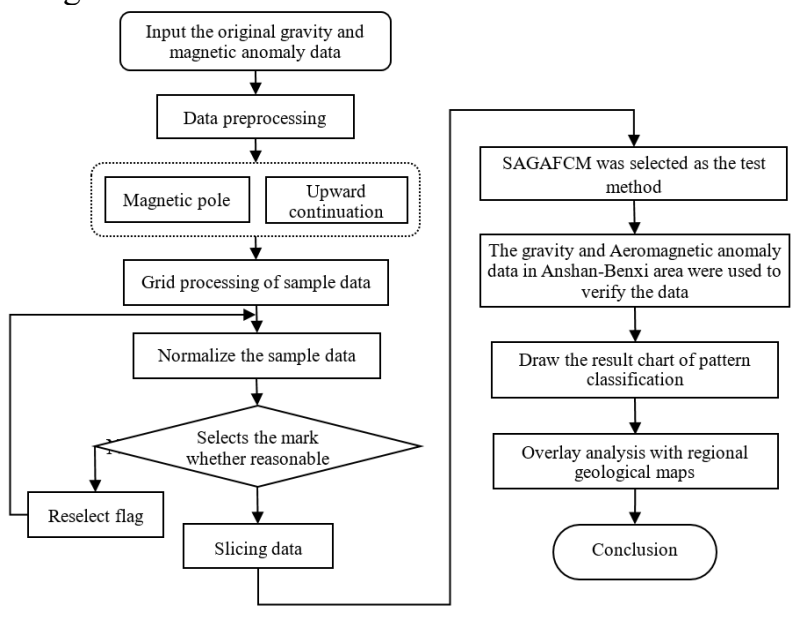

Figure 2. Classification flow chart of magnetic and aeromagnetic data modes

\subsection{Demonstration of Algorithm Application}

The Simulated Annealing (SA) is a heuristic algorithm and an important tool for optimizing non-convex problems. In 1983, Kirkpatrick et al[8]. proposed the SA algorithm and applied it to combinatorial optimization problems successfully. The central idea is: at a certain temperature, the search from one state random change to another state; As the temperature drops continuously to the lowest temperature, the search process stays at the optimal solution with a probability of 1 .

(1) Initialize. A certain value is randomly selected as the initial value $x(0)$ of the variable, and the initial temperature $T(0)$, the termination temperature $T_{\text {final }}$, the temperature drop formula and the corresponding parameters are set.

(2) Run the Metropolis algorithm. A new state $x(n)$ is generated near the current state $x^{\prime}(n)$ by certain rules, and $f(x(n))$ and $f\left(x^{\prime}(n)\right)$ are calculated.

$$
\Delta f=f\left(x^{\prime}(n)\right)-f(x(n))
$$

If $\Delta f<0$ is true, $x^{\prime}(n)$ is superior to $x(n)$, then use $x^{\prime}(n)$ as the value of the next state: $x(n+1)=$ $x^{\prime}(n)$. If $\Delta f>0$ is true, it means that the energy is higher. Do the probability operation. Calculate: $p=$ $e^{-\frac{\Delta f}{T}}$

Then generate a random number $\xi$ from $0 \sim 1$. If $\xi<$ $p$, then accept $x^{\prime}(n)$ as the next state value, otherwise reject $x^{\prime}(n)$, and the next state value remains the same:

$$
x(n+1)=x(n)
$$

(3) According to the internal cycle termination criteria, check whether the heat balance is reached. In the internal cycle, the system iterates at a certain temperature $T$ until the thermal equilibrium condition is met. At this time, the temperature should be modified before the cycle begins. The conditions for the termination of the inner cycle include: whether the value of the cost function tends to be stable, sampling according to a certain number of steps, etc. If it is judged that thermal balance has been reached, go to Step (4); otherwise, go to Step (2) to continue iteration.

(4) Adjust $T$ according to the formula, and check whether the annealing algorithm converges according to the outer cycle termination criterion. If the new temperature value is less than the given termination temperature, then the algorithm will end, and the state $x(n)$ at this point is the optimal condition. Otherwise, go to Step (2) to continue iterating. The criterion for the termination of the outer loop can also be set to a fixed number of iterations, after which the system stops calculation. If the entropy value of the system has reached a minimum, the minimum temperature can be considered to have been reached. Or several times of continuous cooling without improvement of the cost function can also be used as the basis for reaching the termination temperature. Parameters related to temperature $T$ have a great influence on the operation of the SA algorithm. The initial temperature is high enough and the temperature drops slowly enough, so that the system can achieve high quality solution, but the cost of time is also considerable, which needs to be weighed in the practical application. According to the pre-selected marks, the polarized and upward extended data were cut according to the linear interpolation method and used as the experimental input data. The initial input parameter values were set in the improved simulated annealing algorithm. After a hundred iterations, the objective function values generated were shown in Table 2. 
Table 2. SAGAFcm experimental result parameter value list

\begin{tabular}{|c|c|c|c|c|c|c|}
\hline $\begin{array}{l}\text { number of } \\
\text { categories }\end{array}$ & $\begin{array}{c}\text { cooling } \\
\text { factor }\end{array}$ & iterations & $\begin{array}{l}\text { Termination } \\
\text { temperature }\end{array}$ & $\begin{array}{l}\text { Value of objective } \\
\text { function (unextended) } \\
\text { Dandong region }\end{array}$ & $\begin{array}{l}\text { Target function value } \\
\text { (extended up } 8 \mathrm{~km} \text { ) } \\
\text { Dandong region }\end{array}$ & $\begin{array}{l}\text { Value of objective function } \\
\text { (8000 m upward) } \\
\text { Liaoning region }\end{array}$ \\
\hline 4 & & & & 68529059.48 & 28505997.25 & 3981255841.93 \\
\hline 8 & & & & 12828904.52 & 5342659.89 & 883618624.17 \\
\hline 16 & 0.8 & 100 & 99.99 & 2727543.73 & 1112614.16 & 213964934.94 \\
\hline 32 & & & & 635558.45 & 257185.62 & 56228902.96 \\
\hline 64 & & & & 157726.20 & 63050.80 & 13949847.28 \\
\hline
\end{tabular}

\section{Results and Discussion}

SAGAFcm classification is a process of high-order processing of gravity and Aeromagnetic data. Cluster analysis and optimization algorithm are used to organically combine gravity data and aeromagnetic data in order to obtain the combined characteristics of gravity and aeromagnetic field in the study area, so as to explain geological phenomena.

SAGAFcm classification is actually a comprehensive extraction and visualization of geophysical data, so it is extremely sensitive to changes in geophysical data. Thousands of latitude and longitude points in the classification process ensure the accuracy and reliability of the classification. However, due to the fact that geophysical data cannot fully reflect geological phenomena, the limitations of geophysical methods and the multiplicity of interpretation of results will also be reflected in the SAGAFcm classification method.

In general, SAGAFcm classification method has a good identification effect on the boundary between ore body and rock mass, for the following reasons:

Firstly, most metal deposits have obvious gravity and aeromagnetic anomalies, which are significantly different from the surrounding terrane, so the high-resolution gravity and magnetic inversion is easy to identify.

Secondly, most rock bodies are destroyed in the process of magma emplacement. Therefore, the gravity and aeromagnetic data basically reflect the geophysical properties of a single rock mass, excluding the interference of other strata.

\section{Limitations and future work}

The limitations of SAGAFCM are also obvious. The defects of clustering algorithm will also be reflected in SAGAFCM classification method. The main defects include two points:

1. Too high compulsion: cluster analysis will divide the random data into several specified categories, but the possible false positive and false negative will affect the final classification results.

2. The validity of cluster analysis depends on whether the data are separable in nature, but the actual geological phenomena are not all absolutely separable.

Due to the mandatory and norm reference of cluster analysis itself, the dependence on subjective judgment can't be completely ruled out. In practical application, improper data selection and wrong judgment will lead to wrong geological interpretation.

\section{Acknowledgement}

This research was funded by the China Geological Survey, grant number 1212011220247

\section{References}

1. Kirkpatrick S, Gelatt C D, Vecchi A. Optimization by Simulated Annealing[J]. Science, 1983.

2. Peng C, Xue L F, Zhu M, et al. The location and evolution of the tectonic boundary between the Paleoproterozoic Jiao-Liao-Ji Belt and the Longgang Block, northeast China [J]. Precambrian Research, 2016, 272:18-38.

3. Li, S.Z., Yang, Z.S., 1997. Types and genesis of palaeoproterozoic granites in the JiaoLiao Massif. Northwest Geol. 43, 21-27 (in Chinese with English abstract).

4. Zhu, Y.Z., Wang, Y.J., Zhao, J.J., 1982. The stratigraphic position and the age of the early precambrain iron ore Anshan China. Changchun Coll. Geol. S1, 72-83+167 (in Chinese with English abstract)

5. Chun-Hui Xu, Lin-Fu Xue. 2019. The spatial relationship between the development of BIF iron ore in Liaodong area and the Liao-ji rift, Northeast China. Proceedings of the 6th Academic Conference of Geology Resource Management and Sustainable Development[C]. 188-195.

6. Yanpei D, Lianchang Z, Changle W, et al. Genetic type, formation age and tectonic setting of the Waitoushan banded iron formation, Benxi, Liaoning Province [J]. Acta Petrologica Sinica, 2012, 28(11): 3574-3594.

7. ShinsukeNakao, Julie Najita, KenziKarasaki, Hydraulic well testing inversion formodeling fluid 
flow in fractured rocks using simulated annealing: a case study atRaymond field site, California[J], Journal of Applied Geophysics 45(2000). 203-223. 